\title{
Histiocitosis de células de Langerhans pulmonar. Caso clínico
}

\author{
RODRIGO OSSES A. ${ }^{1}$, SERGIO GONZÁLEZ B. ${ }^{2}$, \\ MARCIA AGUIRRE Z. ${ }^{3}$, FERNANDO SALDÍAS P.
}

Enfermedades Respiratorias.

${ }^{2}$ Departamento de

Anatomía Patológica.

Facultad de Medicina,

Pontificia Universidad Católica

de Chile, Santiago de Chile.

${ }^{3}$ Servicio de Medicina, Hospital

Regional de Antofagasta,

Antofagasta, Chile.

Recibido el 4 de mayo de

2010, aceptado el 16 de

diciembre de 2010.

Correspondencia a:

Dr. Fernando Saldías Peñafiel

Departamento de

Enfermedades Respiratorias

Pontificia Universidad Católica

de Chile

Teléfonos: 6331541 - 3543242

Fax: 6335255

Marcoleta 350 - 1er Piso

Santiago, Chile.

Email: fsaldias@med.puc.cl

\section{Adult pulmonary Langerhans cell histiocytosis. Report of one case}

Adult pulmonary Langerhans cell histiocytosis (PLCH) is a rare disorder of unknown etiology that occurs predominantly in young smokers, with an incidence peak at 20-40 years of age. In adults, pulmonary involvement with Langerhans cell histiocytosis usually occurs as a single-organ disease and is characterized by focal Langerhans cell granulomas infiltrating and destroying distal bronchioles. We report a 23-year-old asymptomatic male smoker with a non-specific interstitial infiltrate found in preventive chest $X$ ray examination. A high resolution chest $C T$ scan showed multiple cystic structures predominating in the upper lobes, with small centrilobular nodules. A transbronchial biopsy showed a lymphocytic lung infiltrate with Langerhans cells. A surgical biopsy confirmed the diagnosis of pulmonary Langerhans cell histiocytosis. After six months of follow up, the patient is in good conditions.

(Rev Med Chile 2011; 139: 230-235).

Key words: Dendritic cells; Histiocytosis, Langehaus-cell; Tobacco smoking.

L a histiocitosis de células de Langerhans corresponde a un grupo heterogéneo de enfermedades de origen desconocido, con manifestaciones clínicas y pronóstico variable, caracterizadas por la infiltración de histiocitos anormales en los tejidos, a menudo organizados en granulomas ${ }^{1-3}$. La clasificación propuesta por la Histiocyte Society ${ }^{2}$ está basada fundamentalmente en el número de órganos afectados: a) Enfermedad de Letterer-Siwe: Enfermedad multisistémica aguda de evolución agresiva que predomina en niños pequeños y ocasionalmente en adultos mayores; b) Enfermedad de Hand-Schüller-Christian: Trastorno multifocal que predomina en la infancia y adolescencia, de mejor pronóstico; c) Granuloma eosinófilo: Compromiso de un órgano o sistema (hueso, pulmón o piel), de curso más benigno y que puede regresar espontáneamente ${ }^{1-3}$.

La histiocitosis de células de Langerhans del pulmón (HPCL) es una enfermedad pulmonar difusa poco frecuente de causa desconocida, asociada al consumo de tabaco, caracterizada por la proliferación de células de Langerhans que infiltran y destruyen los bronquiolos respiratorios ${ }^{4-7}$. Su curso clínico es impredecible y se desconoce cuál es la mejor estrategia de seguimiento y manejo de estos pacientes. En relación a un caso clínico revisaremos la epidemiología, etiopatogenia, evaluación funcional, diagnóstico y tratamiento de esta entidad.

\section{Caso clínico}

Hombre de 23 años, fumador de 1-2 cigarrillos/ día, sin comorbilidades, asintomático respiratorio, en control de salud preocupacional se realizó radiografía de tórax que reveló infiltrado intersticial tenue bilateral de carácter inespecífico. No refería compromiso del estado general, sensación febril, astenia, fatigabilidad, disnea, dolor torácico, tos, expectoración ni hemoptisis. No refería exposición a aves, medicamentos ni gases tóxicos. En la infancia fue tratado por glomerulonefritis aguda post-estreptocócica y hepatitis aguda.

En el examen físico destacaba paciente en buenas condiciones generales, mesomorfo, signos vitales normales y $\mathrm{SpO}_{2}$ de $98 \%$ con aire ambiental. 
Bien hidratado, sin lesiones en la piel ni mucosas. No se palpaban adenopatias. No tenía lesiones en cabeza y cuello. Tórax: sin lesiones externas, con expansión simétrica y sonoridad normal, murmullo pulmonar conservado sin ruidos agregados. Examen cardiaco normal. Sin alteraciones en el examen abdominal, no se palparon masas ni visceromegalia. Sin lesiones de extremidades ni esqueleto axial, no presentaba edema ni hipocratismo digital.

En la consulta inicial se realizó tomografía computada de tórax de alta resolución que demostró múltiples estructuras quísticas pequeñas de paredes

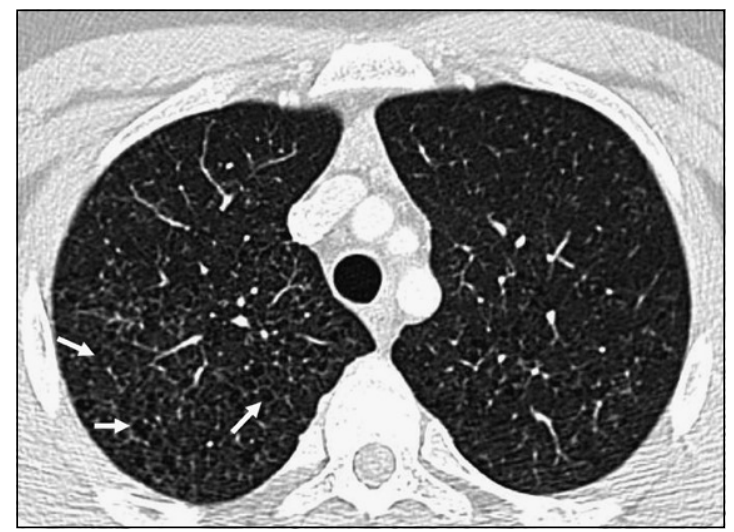

Figura 1. La tomografía computarizada de tórax al momento del diagnóstico muestra múltiples lesiones quísticas de pared delgada (flechas) asociadas a pequeños nódulos de distribución centrolobulillar y predominio en lóbulos superiores.

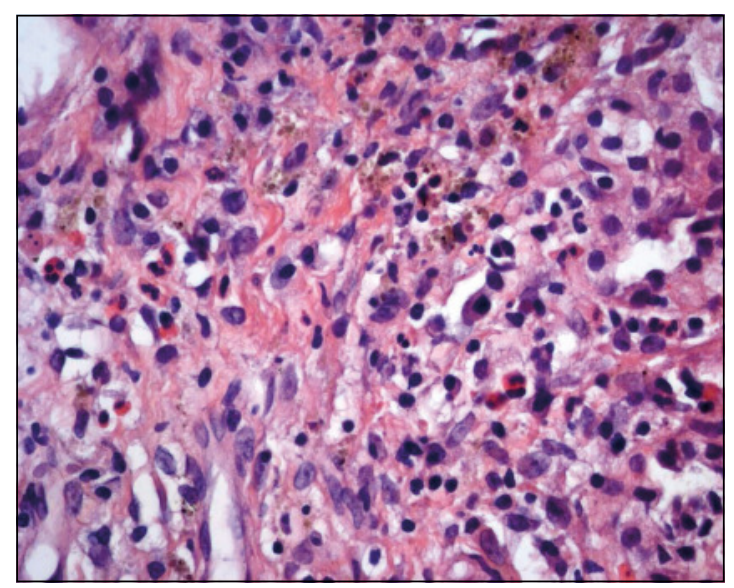

Figura 3. Microfotografía de biopsia transbronquial, que muestra infiltrado de células de Langerhans, eosinófilos y linfocitos pequeños (Hematoxilina eosina $400 \mathrm{X}$ ). mal definidas, con predominio en lóbulos superiores, asociadas a pequeños nódulos de distribución centrolobulillar (Figura 1). No tenía adenopatías mediastínicas ni lesiones óseas o de partes blandas. Se planteó el diagnóstico de histiocitosis pulmonar y se realizó una fibrobroncoscopia, que no demostró alteraciones de la mucosa bronquial, y una biopsia transbronquial que demostró infiltrado pulmonar linfocitario con acúmulos de células de Langerhans (Figuras 2 y 3 ). La tinción inmunohistoquímica con anticuerpos monoclonales mostró reacción positiva para el antígeno $\mathrm{CD} 1 \mathrm{a}$ (Figura 4), lo que apoyó el diagnóstico de HPCL.

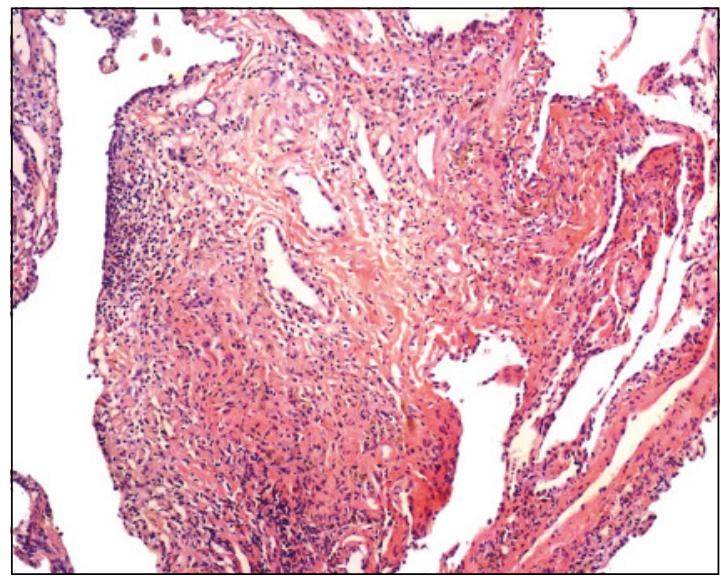

Figura 2. Microfotografía que muestra los tabiques engrosados por fibrosis con silueta estrellada e infiltrado celular mixto de histiocitos y linfocitos (Hematoxilina eosina $100 \mathrm{X}$ ).

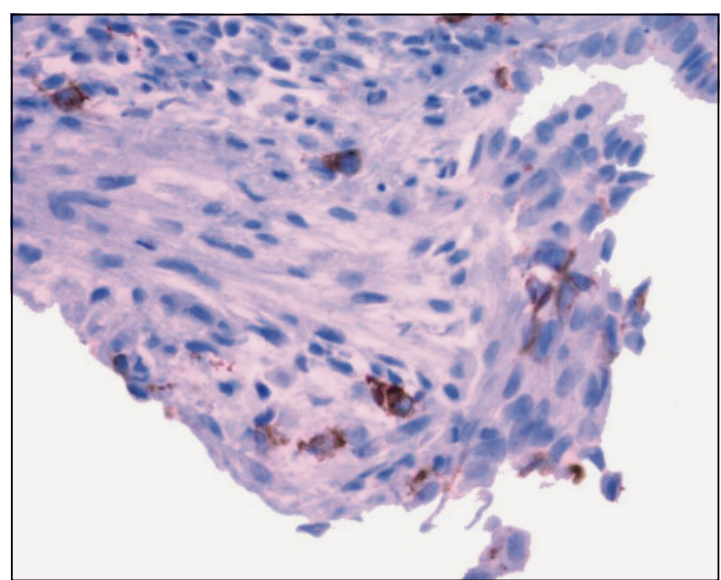

Figura 4. Las células de Langerhans en la tinción inmunohistoquímica fueron positivas para el antígeno CD1a. 
En la Tabla 1 se describen los exámenes de laboratorio y la evaluación funcional respiratoria. No tenía anemia, leucocitosis, VHS elevada, ni alteraciones de la función renal o hepática. La espirometría demostró obstrucción bronquial leve que revierte con el broncodilatador. Los volúmenes pulmonares, DLCO y prueba de caminata de 6 minutos estaban dentro del rango normal.

Se indicó cese inmediato del hábito tabáquico, esteroides inhalados (budesonida $400 \mu \mathrm{g}$ cada 12 horas), evitar realizar actividad laboral en altura por el riesgo de neumotórax y seguimiento trimestral con exámenes de función pulmonar. En el control, el paciente refirió dolor sordo en la región parietal izquierda donde se palpaba aumento de volumen indurado. Se realizó biopsia quirúrgica que confirmó el diagnóstico de HPCL. En el seguimiento a los seis meses, el paciente se mantenía asintomático y sin cambios significativos en la evaluación funcional respiratoria.

\section{Discusión}

La HPCL es una enfermedad intersticial poco prevalente, de causa desconocida, asociada al consumo de tabaco y caracterizada por la proliferación de células de Langerhans que infiltran y forman múltiples nódulos peribronquiolares, bilaterales, los cuales se pueden cavitar y formar lesiones quísticas, con destrucción de grado variable de las estructuras bronquiolares y vasculares ${ }^{4-7}$.

No existen datos precisos acerca de la incidencia de esta enfermedad por dificultades en la pesquisa de los casos oligosintomáticos, falta de registro adecuados y diferentes criterios empleados para establecer el diagnóstico. La HPCL se ha encontrado en menos de 5\% de biopsias pulmonares abiertas realizadas a pacientes con enfermedad pulmonar difusa ${ }^{8}$. En un registro de 3.000 pacientes con enfermedades intersticiales se logró documentar en 2,8\% de los casos en cuatro años de seguimiento ${ }^{1-3}$.

En Japón, Watanabe y cols, encontraron una prevalencia de 0,27 y 0,07 por 100.000 habitantes, en hombres y mujeres, respectivamente ${ }^{9}$. De ellos, más de $90 \%$ eran fumadores, iniciando el consumo de tabaco antes de los 20 años en la mitad de los casos. La enfermedad predomina en el grupo de 20 a 40 años y se presenta en forma esporádica, aunque se han descrito casos familiares ${ }^{4-6,10}$.
Tabla 1. Exámenes de laboratorio y función pulmonar de paciente portador de histiocitosis de células de Langerhans del pulmón

\begin{tabular}{|c|c|c|}
\hline $\begin{array}{l}\text { Exámenes de } \\
\text { laboratorio }\end{array}$ & $\begin{array}{c}\text { Junio } \\
09\end{array}$ & $\begin{array}{l}\text { Octubre } \\
09\end{array}$ \\
\hline Hematocrito (\%) & 37,8 & 43,3 \\
\hline Hemoglobina (g/dL) & 13,1 & 14,6 \\
\hline Leucocitos $\left(x \mathrm{~mm}^{3}\right)$ & 8.100 & 7.900 \\
\hline Eritrosedimentación (mm/h) & 18 & 7 \\
\hline Fosfatasas alcalinas (U/L) & 75 & 87 \\
\hline Albúmina (g/dL) & 4,8 & 4,7 \\
\hline Glucosa (mg/dL) & 86 & 92 \\
\hline Nitrógeno ureico (mg/dL) & 15 & 14 \\
\hline Creatininemia (mg/dL) & 0,77 & 0,69 \\
\hline \multicolumn{3}{|l|}{ Espirometría } \\
\hline CVF (L) & $5,70(112 \%)$ & $6,11(118 \%)$ \\
\hline $\operatorname{VEF}_{1}(\mathrm{~L})$ & $3,86 \quad(88 \%)$ & $4,46(100 \%)$ \\
\hline $\mathrm{VEF}_{1} / \mathrm{CVF}$ & $68 \%$ & $73 \%$ \\
\hline \multicolumn{3}{|l|}{ DLCO } \\
\hline $\mathrm{DLCO}(\mathrm{ml} / \mathrm{min} / \mathrm{mmHg})$ & $29,9 \quad(93 \%)$ & $30,0(93 \%)$ \\
\hline VA (L) & $6,7 \quad(94 \%)$ & $6,8(94 \%)$ \\
\hline DLCO/NA (ml/min/mmHg/L) & $4,5(100 \%)$ & $4,4(98 \%)$ \\
\hline \multicolumn{3}{|l|}{ DR6M } \\
\hline Distancia (m) & $631(75 \%)$ & $644(79 \%)$ \\
\hline $\mathrm{SaO}_{2} \mathrm{R}-\mathrm{E}(\%)$ & $96 \%-98 \%$ & $98 \%-97 \%$ \\
\hline FC R - E (lat/min) & $61-126$ & $68-142$ \\
\hline Disnea $R$ - E & $0-2$ & $0-\quad 1$ \\
\hline
\end{tabular}

Nota: CVF: Capacidad vital forzada, VEF, Volumen espiratorio forzado de $1^{\text {er }}$ segundo, DLCO: Capacidad de difusión de CO, VA: Volumen alveolar, DR6M: Prueba de caminata de 6 minutos, $\mathrm{SaO}_{2}$ : Saturación arterial de oxígeno, FC: Frecuencia cardiaca, R: Reposo, E: Ejercicio. Los valores son expresados como valor absoluto y porcentaje del valor teórico.

Si bien se han descrito casos asociados a neoplasias, tales como leucemias, linfomas, cáncer pulmonar, no se ha demostrado una clara relación ni menos un rol patogénico. Sólo está bien establecida su relación con el hábito de fumar, habitualmente en relación a una carga tabáquica importante, sobre 20 paquetes/año ${ }^{4-7,11}$. En los primeros estudios se reportaba una mayor prevalencia en hombres, observándose un aumento de la incidencia en mujeres, vinculada al aumento del consumo de tabaco ${ }^{4-6,11}$. 
Las células de Langerhans (CL) pertenecen a la familia de las células dendríticas, las cuales forman parte de los mecanismos defensivos del huésped al actuar como presentadoras de antígenos a los linfocitos $\mathrm{T}$ y multiplicadoras de la respuesta inmune, estrechamente ligadas a la función de los macrófagos. Están presentes en órganos linfoides y otros tejidos como la piel, corazón y pulmón ${ }^{4,12}$. Las células dendríticas pulmonares se encuentran en la vaina conectiva broncovascular, intersticio pulmonar, pleura visceral y tejido linfoide asociado al árbol bronquial, y pueden participar en la defensa contra agentes externos iniciando una respuesta inmune tipo Th-1. Además, y no menos importante, es su rol en generar tolerancia a los alergenos inhalados en pulmones de personas normales, sin desencadenar fenómenos inflamatorios ${ }^{4,6,12}$. Las CL se localizan exclusivamente en el epitelio bronquial y bronquiolar donde a través de largas digitaciones forman una extensa red $^{4,13}$. En el citoplasma se reconocen los gránulos de Birbeck (sólo visibles a la microscopia electrónica), organelos específicos relacionados con la internalización de elementos exógenos, así como por la expresión de CD1a y del marcador específico Langerina (CD 207), una lectina asociada constitutivamente a estas estructuras ${ }^{12,13}$

La presentación clínica de la enfermedad es heterogénea, diagnosticándose en distintos escenarios: a) Paciente asintomático, pesquisado por examen radiológico de rutina, lo que ocurre en cerca de 25\% de los casos; b) Cuadro sintomático, que se manifiesta por tos seca y, ocasionalmente, disnea de esfuerzos asociado a síntomas constitucionales (astenia, fiebre, sudoración nocturna, pérdida de peso); ocurre en dos tercios de los pacientes; c) Neumotórax espontáneo, forma de debut en 10-20\% de los pacientes ${ }^{4-9}$.

La mayoría de los pacientes son fumadores, en la tercera o cuarta década de la vida, sin diferencias significativas de género ni raza. El examen físico, de no haber neumotórax, es habitualmente normal, rara vez se describen crepitaciones y, en forma excepcional, hipocratismo digital ${ }^{4-7,11}$. De existir compromiso extrapulmonar, éste es principalmente óseo (10-20\%) caracterizado por lesiones osteolíticas ${ }^{4,7}$ y rara vez se describe diabetes insípida por compromiso infiltrativo de la hipófisis (5\%) o lesiones cutáneas ${ }^{4,7}$.

El neumotórax se presenta en 4-17\% de los pacientes en algún momento de su evolución. En una revisión de 102 casos se describen 16 pacientes que presentaron esta complicación, en dos tercios como manifestación inicial. La recurrencia fue elevada $(\sim 60 \%)$ en los pacientes que no fueron sometidos a pleurodesis ${ }^{4,7,9}$.

Los exámenes generales no muestran hallazgos característicos o específicos de esta entidad. Pese a presentar lesiones radiológicas, $10-15 \%$ de los casos tienen función pulmonar normal ${ }^{4-7}$. Las principales alteraciones funcionales descritas son la disminución de la DLCO (70-90\%), atribuible al engrosamiento de la membrana alveolo-capilar y compromiso vascular pulmonar ${ }^{4,7}$. El patrón espirométrico puede ser normal u obstructivo y la curva flujo volumen es anormal en la mitad de los casos; el patrón obstructivo, raro en enfermedad pulmonar difusa, orienta al diagnóstico cuando está presente $e^{4-7,14-16}$. En los volúmenes pulmonares destaca la disminución de las capacidades vital y pulmonar total (CPT), con aumento del volumen residual (VR) y relación VR/CPT ${ }^{7}$. Los gases en sangre arterial son habitualmente normales o revelan hipoxemia leve en reposo; en el ejercicio puede aparecer hipoxemia y aumento de la diferencia alvéolo-arterial de oxígeno ${ }^{7,14}$.

La radiografía de tórax es anormal en $90 \%$ de los pacientes ${ }^{4,7}$. Los principales hallazgos son infiltrados intersticiales con patrón micronodular o reticulonodular, bilaterales y simétricos, con predominio de zonas medias y superiores de ambos campos pulmonares, con volúmenes normales o aumentados. En la medida que progresa la enfermedad aparecen quistes predominantemente en lóbulos superiores y tienden a desaparecer los nódulos $^{4-7,14,17}$. Ocasionalmente, la radiografía es normal o se observan alteraciones inespecíficas, como imágenes de relleno alveolar, adenopatía hiliar, arterias pulmonares prominentes y derrame pleural asociado a neumotórax ${ }^{4,7,17}$.

La tomografía computada de tórax (HRCT) permite evidenciar las alteraciones propias de la enfermedad. Los principales hallazgos son infiltrados nodulares y lesiones quísticas con predominio en zonas superiores y medias, respetando habitualmente las bases pulmonares. Los nódulos son pequeños, de 1-5 mm de diámetro, aspecto irregular, en número variable y localización centrilobulillar, peribronquial o peribronquiolar. Eventualmente pueden ser cavitados y desaparecer o mostrar regresión con la evolución de la enfermedad. Los quistes de pared delgada, pueden 
variar de forma (ovoidea, sacular, como "hojas de trébol”, etc) y tamaño, pudiendo alcanzar varios centímetros en algunas ocasiones. En la fase inicial predominan los nódulos y los quistes al progresar la enfermedad. La coexistencia de lesiones quísticas bilaterales, de pared fina, asociadas a nódulos pequeños peribronquiolares, con predominio en lóbulos medio y superior, es altamente sugerente de HPCL ${ }^{4-7,17}$. El cuadro clínico-radiográfico clásico permite plantear el diagnóstico obviando la necesidad de una biopsia pulmonar. En la fase inicial se describen alteraciones tomográficas en 5\% de los pacientes con radiografía de tórax normal ${ }^{7,17}$.

La HPCL se caracteriza histológicamente por la presencia de lesiones nodulares bronquiolocéntricas, de aspecto estrellado, conteniendo acúmulos de CL y otras células inflamatorias. Las CL se caracterizan ultraestructuralmente por la presencia de los gránulos de Birbeck y en la inmunohistoquímica son positivas a marcadores como CD1a, proteína S-100, OKT-6 y langerina. Puede asociarse a lesiones propias del tabaquismo, como descamación de macrófagos y elementos de bronquiolitis respiratoria asociada a enfermedad intersticial (RB-ILD), neumonía intersticial descamativa (DIP) y enfisema pulmonar centrolobulillar ${ }^{11}$.

El diagnóstico diferencial corresponde a las enfermedades pulmonares difusas que se manifiestan con infiltrados nodulares y/o quísticos. Las enfermedades de predominio nodular como la sarcoidosis, silicosis, cáncer metastásico, tuberculosis miliar e infecciones con diseminación hematógena constituyen algunos de los más frecuentes. En las lesiones quísticas se debe considerar el enfisema centrilobulillar, linfangioleiomiomatosis, neumonía intersticial linfocitaria, fibrosis quística y esclerosis tuberosa.

El diagnóstico presuntivo se establece considerando los antecedentes clínicos y consumo de tabaco, la extensión y distribución de las lesiones radiográficas y las características funcionales (patrón obstructivo) ${ }^{4-7,17}$. Ante la sospecha clínica o el hallazgo incidental de infiltrados radiológicos que sugieran el diagnóstico, éste se establece con certeza mediante el análisis histopatológico del tejido pulmonar. Éste puede ser obtenido por vía transbronquial o quirúrgica (habitualmente por videotoracoscopia), prefiriéndose esta última debido al mayor tamaño y calidad de la muestra ${ }^{4,7}$.

El curso clínico de la enfermedad es variable, muchos pacientes se mantienen estables por períodos prolongados o presentan regresión espontánea, lo cual dificulta la evaluación del tratamiento. La sobrevida estimada es $74 \%$ a los 5 años y $64 \%$ a los 10 años de evolución, con una sobrevida media de 12,5 a 13 años $^{10,14}$. En el momento del diagnóstico, son factores de mal pronóstico: edad avanzada, disminución de la DLCO y VEF,

En todos los pacientes se recomienda suspender el hábito tabáquico, reportándose ocasionalmente regresión de los infiltrados pulmonares con esta medida ${ }^{18,19}$. El tratamiento esteroidal se reserva para pacientes graves o sintomáticos con enfermedad nodular ${ }^{4-7}$ en los cuales se espera reducir los síntomas constitucionales y respiratorios, así como eventualmente disminuir la progresión de los infiltrados y desarrollo de fibrosis pulmonar. Se recomienda tratar con prednisona o prednisolona en dosis de $0,5-1,0 \mathrm{mg} / \mathrm{kg} /$ día con descenso progresivo de la dosis en 6 a 12 meses. No existen estudios controlados que avalen esta conducta. En la enfermedad multisistémica se ha empleado inmunosupresores (vinblastina, metotrexato o ambos) en combinación con corticoides, sin clara demostración de su eficacia ${ }^{4,7}$. En casos anecdóticos se ha empleado etanercept (anti TNF $\alpha$ ), dexametasona, ciclofosfamida, etopósido, con resultado variable $e^{4,7,20}$.

El trasplante pulmonar se reserva para los enfermos con insuficiencia respiratoria crónica por progresión de la enfermedad fibrótica, refractaria a tratamiento esteroidal e inmunosupresor y en raros casos para pacientes con hipertensión pulmonar severa ${ }^{1,21}$.

\section{Referencias}

1. Howarth DM, Gilchrist GS, Mullan BP, Wiseman GA, Edmonson JH, Schomberg PJ. Langerhans cell histiocytosis: diagnosis, natural history, management, and outcome. Cancer 1999; 85: 2278-90.

2. Aricò M, Girschikofsky M, Généreau T, Klersy C, McClain K, Grois N, et al. Langerhans cell histiocytosis in adults. Report from the International Registry of the Histiocyte Society. Eur J Cancer 2003; 39: 2341-8.

3. Aricò M. Langerhans cell histiocytosis in adults: more questions than answers? Eur J Cancer 2004; 40: 1467-73.

4. Tazi A, Soler P, Hance AJ. Adult pulmonary Langerhans' cell histiocytosis. Thorax 2000; 55: 405-16.

5. Vassallo R, Ryu JH, Colby TV, Hartman T, Limper AH. 
Pulmonary Langerhans'-cell histiocytosis. N Engl J Med 2000; 342: 1969-78.

6. Sundar KM, Gosselin MV, Chung HL, Cahill BC. Pulmonary Langerhans cell histiocytosis: emerging concepts in pathobiology, radiology, and clinical evolution of disease. Chest 2003;123: 1673-83.

7. Tazi, A. Adult pulmonary Langerhans' cell histiocytosis. Eur Respir J 2006; 27: 1272-85.

8. Gaensler E, Carrington C. Open biopsy for chronic diffuse infiltrative lung disease: clinical, roentgenographic, and physiological correlations in 502 patients. Ann Thorac Surg 1980; 30: 411-26.

9. Watanabe R, Tatsumi K, Hashimoto S, Tamakoshi A, Kuriyama T; Respiratory Failure Research Group of Japan. Clinico-epidemiological features of pulmonary histiocytosis X. Intern Med 2001; 40: 998-1003.

10. Delobbe A, Durieu J, Duhamel A, Wallaert B, the Groupe d'Etude en Pathologie Interstitielle de la Société de Pathologie Thoracique du Nord. Determinants of survival in pulmonary Langerhans' cell granulomatosis (histiocytosis X). Eur Respir J 1996; 9: 2002-6.

11. Rao RN, Goodman LR, Tomashefski JF Jr. Smokingrelated interstitial lung disease. Ann Diagn Pathol 2008; 12: 445-57.

12. Lipscomb MF, Masten BJ. Dendritic cells: immune regulators in health and disease. Physiol Rev 2002; 82: 97-130.

13. Knight SC, Burke F, Bedford PA. Dendritic cells, antigen distribution and the initiation of primary immune responses to self and non-self antigens. Semin Cancer Biol
2002; 12: 301-8.

14. Vassallo R, Ryu JH, Schroeder DR, Decker PA, Limper $\mathrm{AH}$. Clinical outcomes of pulmonary Langerhans'-cell histiocytosis in adults. N Engl J Med 2002; 346: 48490.

15. Friedman PJ, Liebow AA, Sokoloff J. Eosinophilic granuloma of lung. Clinical aspects of primary histiocytosis in the adult. Medicine (Baltimore) 1981; 60: 385-96.

16. Crausman RS, Jennings CA, Tuder RM, Ackerson LM, Irvin CG, King TE Jr. Pulmonary histiocytosis X: pulmonary function and exercise pathophysiology. Am J Respir Crit Care Med 1996; 153: 426-35.

17. Abbott GF, Rosado-de-Christenson ML, Franks TJ, Frazier AA, Galvin JR. From the archives of the AFIP: pulmonary Langerhans cell histiocytosis. Radiographics 2004; 24: 821-41.

18. Von Essen S, West W, Sitorius M, Rennard SI. Complete resolution of roentgenographic changes in a patient with pulmonary histiocytosis X. Chest 1990; 98: 765-7.

19. Mogulkoc N, Veral A, Bishop PW, Bayindir U, Pickering CA, Egan JJ. Pulmonary Langerhans' cell histiocytosis: radiologic resolution following smoking cessation. Chest 1999; 115: 1452-5.

20. Henter JI, Karlén J, Calming U, Bernstrand C, Andersson U, Fadeel B. Successful treatment of Langerhans'-cell histiocytosis with etanercept. N Engl J Med 2001; 345: 1577-8.

21. Boehler A. Lung transplantation for cystic lung diseases: lymphangioleiomyomatosis, histiocytosis $\mathrm{x}$, and sarcoidosis. Semin Respir Crit Care Med 2001; 22: 509-16. 